\title{
Changing diagnostic practices: autism spectrum disorder
}

\author{
Marc Woodbury-Smith
}

\begin{abstract}
SUMMARY
In medical practice it is crucial that symptom descriptions are as precise and objective as possible, which psychiatry attempts to achieve through its psychopathological lexicon. The term 'autism spectrum disorder' has now entered psychiatric nosology, but the symptom definitions on which it is based are not robust, potentially making reliable and valid diagnoses a problem. This is further compounded by the spectral nature of the disorder and its lack of clear diagnostic boundaries. To overcome this, there is a need for a psychopathological lexicon of 'social cognition' and a classification system that splits rather than lumps disorders with core difficulties in social interaction.
\end{abstract}

\section{DECLARATION OF INTEREST}

None.

The vicissitudes of diagnostic labelling are perhaps no better evidenced than in psychiatry, as once again we have been preparing for new diagnostic codes. DSM-5 (American Psychiatric Association 2013 ) is now published and ICD-11 is due by 2015. One of the biggest changes that will affect me, as a clinician working in a developmental clinic, is the exclusion of Asperger's disorder (Asperger syndrome) and the creation of a spectrum of the pervasive developmental disorders (PDDs) as 'autism spectrum disorder' (ASD) - the socalled spectralisation of ASD. A category 'social communication disorder' has been added to capture those individuals whose fundamental impairment is one of communication, and who therefore may not have all the characteristics of the 'autism triad'.

Of course, this should come as no great surprise, as subsequent to the landmark paper by Lorna Wing (Wing 1981), the term 'autism spectrum disorder' (Wing 1997) has been in common use for a number of years. Why, then, do I feel so troubled by this term? The answer to this has become increasingly apparent to me since my move to Canada, where I have practised almost exclusively in clinics specialising in the diagnosis and treatment of children and young adults with PDD. Here, I have become aware of the significant increase in the use of the ASD diagnostic label among children with a variety of vulnerabilities in social interaction and communication, and emotional and behavioural disturbance. This is by no means a North American phenomenon, and in my mind it represents a problematic paradigm shift in psychiatric diagnostics. In this reflection I will expound my belief that this 'spectralisation' of ASD represents a missed opportunity to more carefully define these disorders, and I will offer potential solutions to reverse this and move towards more psychopathologically defined PDD subtypes.

\section{Why is the prevalence of PDDs rising?}

Epidemiological surveys suggest that the prevalence of childhood disorders has increased significantly in recent years. This increase is perhaps most striking for the PDDs: until the early 1990s, prevalence estimates were uniformly low and in the region of 3-4 per 10000 children. Since that time, the rate has been increasing, such that the most recent estimates are between 1 in 88 (Centers for Disease Control and Prevention 2012) and 1 in 100 children (Baron-Cohen 2009a). Of course, disorder surveillance and improved diagnostic skills will account for some of this increase. In addition, the preferential use of the ASD label instead of the more general label 'intellectual disability' (or mental retardation) is a universal phenomenon, often due to the service implications of receiving one diagnosis over the other. There are also biological explanations, such as the impact of increasing paternal age on the risk of de novo rare genetic variants (Kong 2012). The prevalence studies themselves are also not without bias. For example, the significant variation in rates between individual states in the USA in the Centers for Disease Control and Prevention's prevalence study highlights the ascertainment bias inherent in the estimated figure (Singer 2012).

These explanations notwithstanding, the creation of a spectrum of PDD has resulted in a shifting of diagnostic boundaries, with the diagnosis now being given to individuals with

\section{REFLECTION}

Marc Woodbury-Smith is an Assistant Professor in the Department of Psychiatry and Behavioural Neurosciences at McMaster University in Ontario, Canada, and a clinician-scientist in the Centre for Applied Genomics at the Hospital for Sick Children in Toronto. He trained in psychiatry in Southampton and Cambridge, UK, and at Yale University School of Medicine's Child Study Center, USA. $\mathrm{He}$ is interested in developmental psychopathology, and the genetics of autism spectrum disorder. Correspondence DrMarc Woodbury-Smith, Department of Psychiatry and Behavioural Neurosciences, McMaster University, Chedoke Site, Evel Building (Room 457). Sanitorium Road, Hamilton, Ontario L8N 3Z5, Canada. Email: woodbur@mcmaster.ca 
relatively milder impairments of interaction and communication. This, too, will have undoubtedly influenced the rising prevalence. It is important to recognise that many such individuals do indeed meet the criteria for one of the PDDs according to ICD-10, DSM-IV or DSM-5 (World Health Organization 1992; American Psychiatric Association 1994, 2013). However, the disorder is still based on vague symptom descriptions rather than psychopathologically founded definitions, thereby allowing the diagnosing clinician a wide degree of subjective interpretation in terms of symptom presence and disorder boundary.

For example, qualitative impairments of social interaction and communication define two of the three categories of the disorder in ICD-10 and DSM-IV, but what does 'qualitative' truly mean? Indeed, the symptoms described, which include, for example, failure to develop peer relationships and failure to use non-verbal communication, all sound very 'quantitative'. In DSM-5 the term 'qualitative' has been replaced by 'persistent', which is clearer in meaning, but still open to interpretation. The third category, 'restricted and ritualistic patterns of behaviour', often takes the form of pursuit of circumscribed interests that are intense or odd in nature. But at what point does an interest become intense, and who decides if an interest is 'odd' or 'normal'? Such symptom descriptions are imprecise and allow the diagnostician significant leeway when deciding which behaviours align with each diagnostic item, and where exactly to draw the line between normality and abnormality. Formalised assessments such as the Autism Diagnostic Interview -Revised (ADI-R; Lord 1994) and the Autism Diagnostic Observation Schedule (ADOS; Lord 2000) do exist, and go some way to address these shortcomings, but nonetheless, a degree of subjective interpretation does remain.

This absence of a truly psychopathologically based diagnostic system for ASD is very disappointing. Schizophrenia, for example, is defined according to very specific mental symptoms, such as thought echo, insertion and broadcast, or primary delusions. Of course, I am not claiming that these are objective, but their definitions are precise and, among mental health professionals, not open to interpretation. Moreover, these symptoms form a hierarchy such that some symptoms (those historically termed 'first rank') are given more diagnostic weight than the 'second rank' symptoms. In contrast, ASD has no such lexicon.

\section{ASD: the 'splitting' v. 'lumping' debate}

Over the years, researchers have made some very astute observations regarding the psychopathology of PDDs, but unfortunately these have never been included in any diagnostic system. For example, the work of Ami Klin and his colleagues on eye tracking (Rice 2012) has very elegantly demonstrated the gaze disorder that is present from a very early age, which is characterised by a lack of orientation to socially salient information in the environment (such as eyes). Furthermore, a lack of joint attention has been consistently demonstrated in children with ASD. Yet neither gaze disturbance nor joint attention are explicitly identified in the diagnostic criteria. Similarly, impairments in empathising and systematising are characteristic of ASD (Baron-Cohen 2009b) and are measurable, yet they are not included in the diagnostic criteria.

If, therefore, ASD is based on loose definitions, as I have proposed, it seems no great surprise that it has evolved into a spectrum. Despite my contention, there are, of course, other arguments that support lumping PDDs into a spectrum. As Lord et al (2012) have demonstrated, much variability exists between both individual clinicians and diagnostic centres for the PDD subtypes they assign, and this disparity principally reflects differences in the clinicians' use of clinical information. Diagnoses within the spectrum were not arbitrary, but at the same time they were not consistent. Therefore, splitting ASD into subtypes based on clinical features may make less sense than adopting the dimensional approach chosen in DSM-5.

However, the 'splitting' rather than 'lumping' argument goes deeper than this. First, there is evidence that different ASD subphenotypes may have different genetic aetiologies. In particular, genetic linkage studies have consistently identified different linkage signals in association with different phenotypes, although admittedly none of these signals has led to gene discovery (for example, Liu 2008). Nonetheless, it does lend support to the idea that the search for biologically driven subphenotypes may not be a futile task.

Second, lumping does seem to prematurely dismiss poorly understood diagnoses such as the childhood disintegrative disorders and disorders that some would argue are clinically distinct, such as Asperger's disorder. The inclusion of a 'social (pragmatic) communication disorder' category, however, is somewhat unusual, considering that removal of subphenotypes was largely based on their lack of external validity. Although I do support its inclusion as a communication subphenotype, I remain concerned that it may become simply synonymous with the old 'not otherwise specified' category. 


\section{What are the options?}

\section{Honing the definitions}

So what are the potential solutions? Fundamentally, a better psychopathological language is needed that more precisely captures a range of social, communicative and behavioural challenges. It is not enough simply to say that a person has qualitative (or persistent) impairments in the ability to form and maintain relationships, as this may mean something different from one clinician to the next. And different people may form more or fewer relationships with others for different reasons. Many of the neuropsychological phenotypes of PDD are, of course, difficult to measure objectively, although ongoing research is attempting to develop novel experimental paradigms to measure and quantify these psychopathological building blocks of the disorder. As discussed above, psychopathology such as abnormalities in eye gaze and joint attention, or impairments of systematising and empathising/mentalising are measurable and should be included in our nosology. The ADOS and other assessment tools give us the opportunity to measure these domains.

Fundamentally, therefore, future nosological revisions must move towards a more precise definition of disorders of social interaction and communication, and not simply broaden the criteria through the incorporation of vague diagnostic descriptions. And the fact that there is evidence from biological research for the validity of the spectrum should not negate this need. Symptom descriptions are fundamental to medical diagnostics, whatever the underlying aetiology.

\section{Pragmatic diagnosis}

Another solution is not to dismiss the idea of splitting rather than lumping. Although DSM-5 may encourage us to use the term ASD, as clinicians we should make certain that we are using the diagnosis that best captures the person's difficulties, whether this be ASD, Asperger's disorder or childhood disintegrative disorder. Of course, the opportunity to do this may be constrained by implications for accessing services with one diagnosis versus another. Consequently, the term ASD may be favoured in certain geographical jurisdictions if it helps to access specialist services.

\section{Shifting focus: from a diagnostic to a functional approach}

Perhaps more importantly, however, as scientists we should continue to attempt to understand what drives the heterogeneity of the spectrum, particularly as this may have preventive, treatment and prognostic implications. Developments in this area will be afforded by endophenotypebased research. Moreover, genetic research offers the possibility of a new top-down approach to understanding ASD phenotypes, through the detailed delineation of phenotypic features occurring in association with specific genetic abnormalities (Abrahams 2008). Perhaps in time ASD will be decomposed into aetiologically driven syndromes. 'Autism' then becomes a symptom rather than a diagnosis.

In clinical terms, much additional information can be gleaned from, for example, psychological assessments, particularly those that measure cognitive strengths and weaknesses, and ideally these should become an integral part of any assessment. In addition to those related to the core social difficulties (for example, the Systemizing and Empathizing Quotients, and the Reading the Mind in the Eyes Test; Baron-Cohen 2005), measures of language, cognition, sensory integration and functioning may all help in moving the focus away from the diagnosis and onto the individual. To this end, adoption of the World Health Organization's International Classification of Functioning (ICF; World Health Organization 2001) as an integral part of assessment will take the emphasis off diagnosis and instead focus on which domains of functioning are affected, so that realistic treatments can be achieved. The ICF focuses not on the diagnosis itself, but on the person's level of functioning, in terms of both bodily functions and the wider social sphere, capturing what they can or cannot do as a result of their disorder. Ultimately, we must do everything in our power to ensure that we have the appropriate information on which to make valid and meaningful treatment decisions.

\section{References}

Abrahams B, Geshwind D (2008) Advances in autism genetics: on the threshold of a new neurobiology. Nature Reviews Genetics, 9: 341-56.

American Psychiatric Association (1994) Diagnostic and Statistical Manual of Mental Disorders (4th edn) (DSM-IV). APA.

American Psychiatric Association (2013) Diagnostic and Statistical Manual of Mental Disorders (5th edn) (DSM-5). APA.

Baron-Cohen S, Wheelwright S, Lawson J, et al (2005) Empathizing and systemizing in autism spectrum conditions. In Handbook of Autism and Pervasive Developmental Disorders (vol 1) (3rd edn) (eds F Volkmar, R Paul, A Klin, et al): 628-39. John Wiley \& Sons.

Baron-Cohen S, Scott FJ, Allison C, et al (2009a) Prevalence of autismspectrum conditions: UK school-based population study. British Journal of Psychiatry, 194: 500-9.

Baron-Cohen S (2009b) Autism: the empathizing-systemizing (E-S) theory. Annals of the New York Academy of Sciences, 1156: 68-80.

Centers for Disease Control and Prevention (2012) Prevalence of autism spectrum disorders - Autism and Developmental Disabilities Monitoring Network, 14 sites, United States, 2008. Surveillance Summaries, 61(3): $1-19$ 
Kong A, Frigge ML, Masson G, et al (2012) Rate of de novo mutations and the importance of father's age to disease risk. Nature, 488: 471-5.

Liu X, Paterson A, Szatmari P, et al (2008) Genome-wide linkage analyses of quantitative and categorical autism subphenotypes. Biological Psychiatry, 64: 561-70.

Lord C, Rutter M, Le Couteur A (1994) Autism Diagnostic Interview: Revised: a revised version of a diagnostic interview for caregivers of individuals with possible pervasive developmental disorders. Journal of Autism and Developmental Disorders, 24: 659-85.

Lord C, Risi S, Lambrecht L, et al (2000) The Autism Diagnostic Observation Schedule: Generic: a standard measure of social and communication deficits associated with the spectrum of autism. Journal of Autism and Developmental Disorders, 30: 205-23.

Lord C, Petkove E, Hus V, et al (2012) A multisite study of the clinical diagnosis of different autism spectrum disorders. Archives of General Psychiatry, 69: 306-13.
Rice K, Moriuchi JM, Jones W, et al (2012) Parsing heterogeneity in autism spectrum disorders: visual scanning of dynamic social scenes in school-aged children. Journal of the American Academy of Child and Adolescent Psychiatry, 51: 238-48.

Singer E/Fombonne E (2012) 0\&A with Eric Fombonne: Are autism's rising rates real? Simons Foundation (http://sfari.org/news-and-opinion/qa/2012/q-a-with-eric-fombonne-are-autisms-rising-rates-real). Accessed 11 Sep 2013

Wing L (1981) Asperger's syndrome: a clinical account. Psychological Medicine, 11: 115-29

Wing L (1997) The history of ideas on autism. Autism, 1: 13-23.

World Health Organization (1992) The ICD-10 Classification of Mental and Behavioural Disorders: Clinical Descriptions and Diagnostic Guidelines. $\mathrm{WHO}$.

World Health Organization (2001) International Classification of Functioning, Disability and Health (ICF). WHO. 